\title{
Projection Pursuit Flood Disaster Classification Assessment Method Based on Multi-Swarm Cooperative Particle Swarm Optimization
}

\author{
Wei Huang ${ }^{1,2}$, Xingnan Zhang ${ }^{1,2}$ \\ ${ }^{1}$ National Engineering Research Center of Water Resources Efficient Utilization and Engineering Safety, \\ Hohai University, Nanjing, China \\ ${ }^{2}$ College of Hydrology and Water Resources, Hohai University, Nanjing, China \\ E-mail:wei.huang923@gmail.com \\ Received April 8, 2011; revised May 7, 2011; accepted June 6, 2011
}

\begin{abstract}
The indicators of flood damage assessment in the flood classification are often incompatible, and it is very difficult to use those indicators value directly for classification assessment. Projection pursuit technology can project higher dimensional incompatible data into lower dimensional sub-space, and find the projection values for optimal projection index function to get the higher dimensional data structure features, which has been improved to be reasonable and effective for flood disaster classification assessment. However, it is a bit difficult to optimize the parameters of projection index functions, as a result, that limits the applications of this method. As an emerging heuristic global optimization algorithm based on swarm intelligence, particle swarm optimization algorithm has the ability of solving complex optimization problem, but it still be easily convergent early, and can not search the global optimal solution. In this paper, a flood disaster classification assessment method based on multi-swarm cooperative particle swarm optimization is proposed, which adopts a tri-parameter Logistic curve to construct the flood disaster projection pursuit model, and uses multi-swarm system particle swarm optimization method to optimize the parameters of the projection index functions. The typical test function experiment shows that this optimization method can solve the early convergence commonly found in standard particle swarm optimization algorithm, which global optimized ability is improved greatly. Applied in flood disaster assessment in Henan Province, the results using this method comparing with others indicates that it can assess effectively the flood disaster, and has better assessment accuracy and disaster resolution.
\end{abstract}

Keywords: Flood Classification, Particle Swarm Optimization, Projection Pursuit

\section{Introduction}

Flood disaster is one of the natural calamities which have the greatest effect, the highest frequency and the most severe loss [1]. Because of the high dimension and complexity of the flood disaster system, there are no uniform flood disaster assessment indices and technical methods, and flood disaster assessment is still one of the difficult and hot spots in flood disaster research. Flood disaster assessment is to search the non-linear relationship between flood disaster assessment indices and flood disaster grads on the basis of having obtained some disaster assessment indices, so that it can assess the loss degree caused by the flood disaster, which is a pattern recogni- tion problem essentially. In order to estimate the flood disaster objectively and accurately, multi-factor assessment model need to be built. In terms of the flood disaster classification assessment, several methods, such as matter-element analysis method, fuzzy synthetic evaluation method, gray cluster, artificial neural network, and immune genetic algorithm, have been proposed [2-6]. However, the results estimated by those models are discrete disaster degrees, and the disaster resolution is low. The flood disaster assessment model based on projection pursuit technology solves the incompatible problem of flood disaster assessment indices, and the calculative process is simple, while the assessment results are intuitive, steady without artificial random. So this model at- 
taches more and more importance in flood disaster classification [7-8]. Projection pursuit is to project higher dimensional data into lower dimensional sub-space, then, it analyzes the structure characteristic of higher dimensional data and predicts the system output, thus, parameter optimization of projection index function is the key factors whether projection pursuit technology can be applied successfully. However, parameter optimization of projection index function is generally complex which limits the application of this technology. As an emerging heuristic global optimization algorithm based on swarm intelligence, particle swarm optimization algorithm has advantages of simple principle, low controlled parameters, fast convergent rate, which applied widely in fields such as function optimization, PID control, and it becomes one of the fastest developing intelligent optimization algorithm[9-11]. Particle swarm optimization algorithm has the ability of solving complex optimization problem, but for some complex optimization problem, this algorithm is easily convergent early, and can not find out the global optimal solution.

This paper proposes a flood disaster classification assessment method based on multi-swarm cooperative particle swarm optimization. Multi-swarm cooperative particle swarm optimization algorithm has the advantage of information sharing between principal swarm and subordinate swarm, subordinate swarm and subordinate swarm. This algorithm conquers the early convergency problem of ordinary particle swarm optimization algorithm, which improves the global optimizing ability, the optimization effect of projection index function parameters and flood disaster classification assessment precision. In order to validate the rationality and effectiveness, this paper simulates the instance combining flood disaster grades sample data set of Henan Province.

\section{Materials and Methods}

\subsection{Flood Disaster Classification Model Based on Projection Pursuit}

Projection pursuit is one kind of statistic method dealing with multi-factor complex problem, by projecting higher dimensional data into lower dimensional (1D to 3D) sub-space [12]. For projected configurations, projection index function (objective function) is adopted to weigh the possibility of some structures exposed by projection, and finds out the projection value for optimal projection index function, which can reflect higher dimensional data structure or character. Then, the structure character of higher dimensional data is analyzed with this projection value, or the mathematical model is built to predict the system output using the scatter diagram, where, the structure of the projection index function and its optimization problem are the key factors whether project pursuit method can be applied successfully.

The modeling procedure of projection pursuit flood disaster classification model as following:

1) The normalization process of flood disaster index In this paper, we assume that flood sample set is $\{x(i, j) i=1,2, \cdots, n ; j=1,2, \cdots, p\}$, where, $x(i, j)$ is the $i$ th flood and the $j$ th flood disaster index value, $n$ and $p$ is the numbers of flood times and flood disaster index respectively. In order to eliminate dimension of each index value and unify the range of each index value, the below formula is applied for extremal normalization processing:

$$
x^{*}(i, j)=[x(i, j)-E x(j)] / S x(j)
$$

Where, $\operatorname{Ex}(j)$ and $S x(j)$ is the mean value and the mean square value of the $j$ th dimension flood disaster index value, $x^{*}(i, j)$ is the sequence of index eigenvalue normalization.

2) Construction of projection index function

The essential of projection pursuit method is to synthesize $p$-dimensional data $\left\{x^{*}(i, j) \mid j=1,2, \cdots, p\right\}$ to one-dimensional value $z_{i}$ of projection direction.

$$
z_{i}=\sum_{j=1}^{p} a_{j} x^{*}(i, j)(i=1,2, \cdots, n) \quad a_{j} \in[-1,1]
$$

when synthesizing projection value, the distribution characteristics of projection value $z_{i}$ should be (as follows): local projection points are as closer as possible, and agglomerated to several clouds, but global projection points are scattering. Thus, projection index function can be constructed as follows:

$$
Q_{a}=s_{a} \cdot d_{a}
$$

where, $S_{a}$ is the distance function between clusters, which equars the standard deviation of projection value $z_{i} \cdot d_{a}$ is the density function in clusters, which means the local density of projection value $z_{i}$.

$$
\begin{gathered}
s_{a}=\left[\sum_{i=1}^{n}\left(z_{i}-\bar{z}\right)^{2} /(n-1)\right]^{1 / 2} \\
d_{a}=\sum_{i=1}^{n} \sum_{j=1}^{n}\left(R-r_{i j}\right) f\left(R-r_{i j}\right)
\end{gathered}
$$

where, $\bar{z}=\sum_{i=1}^{n} z_{i} / n, r_{i j}=\left|z_{i}-z_{j}\right|(i, j=1,2, \cdots n)$, $R=0.1 S_{a}$

$R$ is the window radius of density function in classes, which is related to data characteristics, while the selection of average number of window projection points should not be too small, and the average deviation of glide should not be too large. $f\left(R-r_{i j}\right)$ is monotonic density function, when $R>r_{i j}, f\left(R-r_{i j}\right)=1$, vice versa, $f\left(R-r_{i j}\right)=0$.

3) Projection index function optimization 
Once flood disaster samples are determined, $Q_{a}$ can only vary with projection direction $a_{j}(j=1,2, \cdots, p)$. Different projection directions reflect different data structure features; the best projection direction is the projection direction that can expose certain feature structure of higher dimensional data. Therefore, the best projection direction can be determined by solving the maximum problem of projection index function, and the flood disaster classification can be obtained by corresponding $z_{i}(i=1,2, \cdots, n)$. Thus, the flood disaster classification problem evolves to an optimal problem solving the best projection direction $a_{j}(j=1,2, \cdots, p)$.

This optimal model is described as follows:

$$
\left\{\begin{array}{l}
\max Q_{a}=s_{a} d_{a} \\
\text { s.t. } \sum_{\mathrm{j}=1}^{p} a_{j}^{2}=1
\end{array}\right.
$$

Because the model optimization is a complex non-linear optimal problem, it is a bit difficult to deal with. In this paper, we will propose a new type of multi-swarm cooperative particle swarm optimization algorithm to solve the flood disaster classification problem.

4) Flood disaster classification

The best projection direction obtained by above steps is inserted into projection value $z_{i}$ corresponding to the $i$ th flood disaster, and the mathematical model is created according to the scatter diagram of $z_{i}$ and flood disaster criteria $y_{i}$. On the basis of considering the effect of increasing index of $z_{i}$ to $y_{i}$, the flood disaster assessment model building by a Logistic curve of tri-parameter achieves a good results [13].

$$
y^{*}(i)=\frac{N}{1+e^{C_{1}-C_{2}{ }^{*} \operatorname{Sgn}\left(z^{*}(i) *\right)\left|z^{*}(i)\right|^{C_{3}}}}
$$

Where, $y^{*}(i)$ is the computational value of flood grades of the $i$ th flood disaster, the largest grade $N$ is the upper limit value, $c_{1}, c_{2}$ and $c_{3}$ are undecided parameters, which denotes integrated constant number and increasing rate respectively, and their values are determined by solving the minimum problem below.

$$
\min F\left(c_{1}, c_{2}, c_{3}\right)=\sum_{i=1}^{n}\left(y^{*}(i)-y(i)\right)^{2}
$$

Likewise, the minimum problem also can be determined by particle swarm optimization algorithm. In this paper, a multi-swarm cooperative particle swarm optimization algorithm is proposed.

\subsection{Multi-swarm Cooperative Particle Swarm Optimization Algorithm}

The particle swarm optimization (PSO) algorithm is an optimal method based on swarm evolution proposed by Kennedy et al. in 1995, which has the following advantages: simple principle, little controlled parameters, fast convergent rate and so on [14]. This algorithm is applied in the fields such as function optimization, neural network training, PID control, etc., which gets good optimal affects, and becomes a research hotspot of international evolution computational field. Compared to other algorithms using evolution operators to individuals, PSO algorithm is a kind of swarm intelligent evolution algorithm, which regards each individual as a non-volume particle in D-dimensional searching space, according to the fitness to the environment, individuals in swarm are moved to good fields that flying in a certain velocity in searching space, where the velocity is dynamically adjusted by flying experiences of itself and companions. The location of the $i$ th particle in D-dimensional space is denoted as $Y_{i}=\left(y_{i 1}, y_{i 2}, \cdots, y_{i D}\right)$, and the best location experienced (i.e., the best fitness) is denoted as $P_{i}=\left(p_{i 1}, p_{i 2}, \cdots, p_{i D}\right)$, which is also called $P_{b e s t}$. The best location experienced by all the particles in swarm is recorded as $P_{g}$, which is also named as $G_{\text {best }}$. The velocity of the particle $i$ is denoted by $V_{i}=\left(v_{i 1}, v_{i 2}, \cdots, v_{i D}\right)$. For the particles of each generation, the $d$ dimension $(1<d<D)$ varies with the equation below:

$$
\begin{gathered}
f(x)=\sum_{i=1}^{d}\left(x_{i}^{2}-10 \cos \left(2 \pi x_{i}\right)+10\right) \\
\omega=\omega_{\max }-k \frac{\omega_{\max }-\omega_{\min }}{k_{\max }} \\
y_{i d}^{k+1}=y_{i d}^{k}+v_{i d}^{k+1}
\end{gathered}
$$

Where, $c_{1}$ and $c_{2}$ are accelerated factors, $\operatorname{rand}()$ is the random number in $[0,1], k$ is current iteration time, $v_{i d} \in\left[-v_{i \min }, v_{i \max }\right], \omega$ is the inertial weighed coefficient. Particle swarm algorithm solves the problem by updating iteration of Equation (9) and (10) until getting the satisfactory solution or the largest iteration time.

As a random global optimal algorithm, PSO algorithm has its own limitation [15]. In the initial optimal stage, PSO algorithm has a fast convergent rate, but with the continuing optimal steps, it easily gets into local optimization in later searching stage. The scholars proposed many improved methods for this problem. This paper adopts a new type of particle swarm optimal algorithm based on symbiosis in biology, which is multi-swarm cooperative particle swarm optimizer (MCPSO) [16-17]. This algorithm divides swarm into several sub-swarms, which uses a master-slave structure to simulate symbiosis in sub-swarms and communicates messages in master-slave swarms. Therefore, it avoids the danger of getting into local optimization caused by miscarriage of justice of individuals' information. At the same time, all of the swarms' information communicateons, which re- 
alized by the central particle's guidance determined by the best particle's average location in the whole swarms, communicate information between main swarm and subordinate swarm, meanwhile, the information communications existing in subordinate swarms improve the algorithm's optimal effect. Comparing with the basic PSO algo-rithm, the velocity upgrading equation of MCPSO is

$$
\begin{aligned}
v_{i d}^{k+1}= & c_{1} \cdot \operatorname{rand} \cdot\left(p_{i p}-y_{i d}\right)+c_{2} \cdot \operatorname{rand} \cdot\left(p_{i g}-y_{i d}\right) \\
& +c_{3} \cdot \operatorname{rand} \cdot\left(p_{i c}-y_{i d}\right)+\omega \cdot v_{i d}^{k}
\end{aligned}
$$

Where, $p_{i p}$ and $p_{i g}$ denotes the best location of the particle found by itself and the swarm, $p_{i c}$ is the central location in the whole sub-swarms. When $N$ sub-swarms independently upgrade their velocities and locations, the best location in each sub-swarm is determined, and the equation is as following:

$$
p_{i p}{ }^{(t+1)}=\frac{1}{N} \sum_{i=1}^{n} P_{t g}^{t}
$$

Where, $n=1,2, \cdots, N$, and central particle has no velocity compared with others.

In order to test the optimal performance of MCPSO proposed in this paper, two classical functions (Sphere and Rastrigin) are calculated. Function Sphere is a simple unimodal function, which is used to research the effect of optimal algorithm in problem dimensionality, and its expression is

$$
f(x)=\sum_{i=1}^{n} x_{i}^{2}
$$

The global optimal point of the function locates in $x=\{0,0, \cdots, 0\}$, and the function value of global optimal point is $f(x)=0$. Function Rastrigin is a classical multi-modal function, and is one of the standard problems optimizing algorithm performance test. The expression of Rastrigin function is

$$
f(x)=\sum_{i=1}^{d}\left(x_{i}^{2}-10 \cos \left(2 \pi x_{i}\right)+10\right)
$$

This function has innumerable minimal points, and the global optimal point is hard to find out, where, there is only one minimal point, i.e., when $x=\{0,0\}$, and the global optimal point is $f(x)=0$.

In order to prove the effectiveness of the proposed algorithm, it is compared with the results of standard particle swarm optimal algorithm. The algorithm parameters are set as follows: The dimensionality of test function is 30 , the swarm scale is 80 , where the swarm number in MCPSO algorithm is 4 , and each size of the swarms is $20, c_{1}=c_{2}=2.0, \omega$ is self-adaptively adjusted: $\omega=\omega_{\max }-k \frac{\omega_{\max }-\omega_{\min }}{k_{\max }}$, where, $\omega_{\max }=0.9, \omega_{\min }=0.1$, $k_{\max }$ is the maximal iteration times which equals to 1000 . The two algorithms is repeatedly calculated for 50 times, and the results of the two algorithms in the two classical function tests are shown in Table 1.

The Table1 above indicates that the MCPSO algorithm can avoid early convergence effectively, and after 50-time repeated experiments, the average best adaptive value obtained by MCPSO is better than that of SPSO algorithm, while the standard deviation is small, which mean the algorithm has better abilities of global optimizing and robust.

\section{Results and Discussion}

This paper studies the case of flood disaster grades sample dataset of HeNan Province in China provided in the reference [18], where the flood disaster grades standard of HeNan Province is shown in Table 2, and the flood disaster index data is shown in Table 3. MCPSO-CC parameters are set as follows: the number of sub-swarm is 4 , the size of each sub-swarm is $20, k_{\max }=1000$, and $c_{1}=c_{2}=c_{3}=1.367$

The data in Table 3 is normalized to get $x^{*}(i, j)$ which is inserted into equations (2) to (5), then, projection index functions are obtained which are optimized by MCPSO to get eigenvector $a^{*}=(0.8362,0.5619)$. By inserting $a^{*}$ into equation (2), the flood disaster projection value $z_{i}$ can be got, and the details are shown in Table 3. The relationship between values of $z_{i}$ and $y_{i}$ is expressed by Equation (7), where $N=4, C_{1}, C_{2}$ and $C_{3}$ can be obtained from optimal equation (8) of MCPSO. At last, the projection pursuit model of flood disaster grades assessment of HeNan Province is shown as follows:

Table 1. Two different algorithms for related test results of the test functions.

\begin{tabular}{ccccc}
\hline \multirow{2}{*}{ Algorithm } & \multicolumn{2}{c}{ Sphere } & \multicolumn{2}{c}{ Rastrigin } \\
\cline { 2 - 5 } & Mean & Std & Mean & Std \\
\hline SPSO & $7.3 \mathrm{E}-28 \mathrm{E}$ & $7.0 \mathrm{E}-28$ & 3.4 & 1.5 \\
MCPSO & $1.5 \mathrm{E}-196 \mathrm{E}$ & 0 & $2.7 \mathrm{E}-15 \mathrm{E}$ & $8.8 \mathrm{E}-15 \mathrm{E}$ \\
\hline
\end{tabular}

Table 2. The flood disaster grades standard of HeNan Province.

\begin{tabular}{lcccc}
\hline $\begin{array}{c}\text { Disaster } \\
\text { Index }\end{array}$ & Normal & Medium & Large & Huge \\
\hline $\begin{array}{c}\text { Disaster area } \\
\left(\mathrm{km}^{2}\right)\end{array}$ & $<46.7$ & $46.7-136.7$ & $136.7-283.3$ & $>283.3$ \\
$\begin{array}{l}\text { Direct } \\
\text { economic loss } \\
\text { (Billion US } \\
\text { Dollar) }\end{array}$ & $<0.13$ & $0.13-0.44$ & $0.44-1.21$ & $>1.21$ \\
\hline
\end{tabular}




$$
y^{*}(i)=\frac{N}{1+\left.\left.e^{-1.12-1.5^{*} \operatorname{Sgn}}\left(z^{*}(i)\right)^{*}\right|^{*}(i)\right|^{1.9}}
$$

In order to verify the rationality and the effect of the proposed method, the flood disaster grades assessment of actual 9-time flood from 1950 to 1990 of HeNan Province is evaluated, and the results are shown in Table 3. The same case is simulated by standard particle swarm algorithm (SPSO) and the CCPSO method [13], while the error analysis of calculated results got by the method proposed in this paper are done, and the results of different methods are shown in Table 4.

From Table 4, comparing with method SPSO and CCPSO, the assessment results of proposed MCPSO method has the optimal disaster grade accuracy, high disaster grade resolution. It provides a new way for flood disaster classification.

\section{Conclusions}

Flood disaster classification is used for evaluating the destroyed degree, which based on flood disaster assessment index value and disaster assessment index architecture. Each flood disaster assessment index is incompatible, so it is very difficult to evaluate disaster using the index values. Projection pursuit technology has advantages that projecting higher dimensional incompatible data into lower dimensional sub-space, then obtaining higher dimensional data structure characteristics by searching the optimal projection value of projection index function, which have been improved to be rational and effective in flood disaster classification assessment. However, the complexity of parameter optimal problem of projection index function has become the bottleneck of its development. Although particle swarm optimal algorithm has the ability of solving the parameter optimal problem, it still has the disadvantage of early convergence and can not find the global optimization. This paper proposes a new flood disaster assessment method based on multi-swarm cooperative particle swarm optimization, which constructs a flood disaster assessment projection pursuit model using a tri-parameter Logistic curve, and optimizes the parameters of projection index function using multi-swarm system particle swarm optimization method. The classical test function experiment indicates that this optimization method can effectively overcome early convergence problem existing commonly in standard particle swarm optimization method, and improve the global optimizing ability. The method is applied in case study and compared with others, which indicates this proposed method has better assessment accuracy and disaster grade resolution, meanwhile, it has better application prospect in flood disaster assessment models.
Table 3. Flood disaster index and its assessment results.

\begin{tabular}{cccccc}
\hline NO & \multicolumn{2}{c}{ Disaster index } & Projection & \multicolumn{2}{c}{ Flood disaster grade } \\
\hline$i$ & $X(i, 1)$ & $X(i, 1)$ & $z_{i}$ & $\begin{array}{c}\text { Experien-ce } \\
\text { gradculated }\end{array}$ \\
\hline 1 & 38.7 & 7.9 & -1.168 & 1 & 1.1588 \\
2 & 38.5 & 7.8 & -1.170 & 1 & 1.1544 \\
3 & 32.10 & 6.5 & -1.205 & 1 & 1.0601 \\
4 & 24.2 & 4.9 & -1.249 & 1 & 0.9477 \\
5 & 36.4 & 7.4 & -1.181 & 1 & 1.1238 \\
6 & 46.7 & 9.5 & -1.124 & 1.5 & 1.279 \\
7 & 97.6 & 21.7 & -0.827 & 2 & 2.0733 \\
8 & 60.4 & 12.8 & -1.044 & 2 & 1.5014 \\
9 & 112.6 & 25.2 & -0.740 & 2 & 2.2703 \\
10 & 56.2 & 11.8 & -1.042 & 2 & 1.5058 \\
11 & 80.6 & 17.6 & -0.926 & 2 & 1.8219 \\
12 & 136.7 & 31 & -0.599 & 2.5 & 2.5384 \\
13 & 259.1 & 76.1 & 0.245 & 3 & 3.0914 \\
14 & 200.1 & 54.4 & -0.161 & 3 & 2.9808 \\
15 & 280.1 & 83.8 & 0.390 & 3 & 3.1906 \\
16 & 236.1 & 67.6 & 0.086 & 3 & 3.0266 \\
17 & 157.3 & 38.6 & -0.457 & 3 & 2.7433 \\
18 & 283.3 & 85 & 0.413 & 3.5 & 3.2085 \\
19 & 556.9 & 167.1 & 2.147 & 4 & 3.9979 \\
20 & 649.9 & 194.9 & 2.736 & 4 & 3.9999 \\
21 & 602.3 & 180.7 & 2.435 & 4 & 3.9996 \\
22 & 446.5 & 134 & 1.448 & 4 & 3.938 \\
23 & 694.9 & 208.5 & 3.022 & 4 & 4 \\
1950 & 72.92 & 9.9 & -1.020 & 2 & 1.5678 \\
1954 & 148.13 & 20.65 & -0.642 & 2 & 2.5458 \\
1956 & 203.92 & 27.52 & -0.370 & 3 & 2.8818 \\
1957 & 179.1 & 24.85 & -0.488 & 3 & 2.764 \\
1963 & 375.46 & 94.92 & 0.849 & 4 & 3.5847 \\
1964 & 301.24 & 47.83 & 0.172 & 3 & 3.0611 \\
1975 & 141.97 & 116.43 & 0.132 & 3 & 3.0487 \\
1982 & 279.84 & 121.12 & 0.700 & 4 & 3.4475 \\
1984 & 172.06 & 51.61 & -0.292 & 3 & 2.9398 \\
\hline & & & & &
\end{tabular}

Table 4. Error analysis of disaster grade assessment value of different assessment methods

\begin{tabular}{|c|c|c|c|c|}
\hline \multirow[t]{2}{*}{ Data } & thod & PSO & CCPSO & MCPSO \\
\hline & {$[0,0.1]$} & 39.13 & 43.48 & 52.17 \\
\hline \multirow{4}{*}{$\begin{array}{c}\text { The ratio of } \\
\text { absolute disaster } \\
\text { grade error } \\
\text { falling onto } \\
\text { intervals }(\%)\end{array}$} & {$[0,0.2]$} & 60.87 & 69.57 & 73.9 \\
\hline & {$[0,0.3]$} & 91.30 & 86.96 & 91.30 \\
\hline & {$[0,0.4]$} & 91.30 & 91.30 & 91.30 \\
\hline & {$[0,0.5]$} & 100 & 100 & 100 \\
\hline \multicolumn{2}{|c|}{$\begin{array}{c}\text { Absolute error average value } \\
\text { of disaster grade }\end{array}$} & 0.153 & 0.148 & 0.145 \\
\hline \multicolumn{2}{|c|}{$\begin{array}{c}\text { Relative error average value of } \\
\text { disaster grade }(\%)\end{array}$} & 8.86 & 8.44 & 7.8 \\
\hline
\end{tabular}


Although the constructed model has been simulated and verified, due to many impact factors of flood disaster assessment, non-unique flood disaster grade standards, it should be improved and validated in the future actual applications.

\section{Acknowledgments}

This work is supported by the National Nature Science Foundation of China (No.50879017) and the National Key Technology R\&D Program (2008BAB29B08-02).

\section{References}

[1] E. J. Plate, "Flood Risk and Flood Management," Journal of Hydrology, Vol. 267, No.1-2, 2002, pp. 2-11. doi:10.1016/S0022-1694(02)00135-X

[2] B.-H. Wang and X.-Y. Yang, "The Application of PPC Model of RAGA on the Compositive Evaluation of Flood Disaster in Heilongjiang Province," Journal of Guangdong Technical College of Water Resources and Electric Engineering, 2009, pp. 46-49.

[3] A.-X. Zhao and Z.-J. Ma, "Appraising Study for the Loss Evaluation System of Natural Disasters," Journal of Natural Disasters, 1993, Vol. 2, pp. 1-7.

[4] Y.-Y. He, J.-Z. Zhou, Qin Hui, L. Mo and P.-G. Kou, "Flood Disaster Classification Based on Fuzzy Clustering Iterative Model and Modified Differential Evolution Algorithm," Proceedings of the 6th International Conference on Fuzzy Systems and Knowledge Discovery, Tianjing, 14-16 August 2009, Vol 3.

[5] Y.-N. Chen and S.-Q. Yang, "Application of Grey Clustering Analysis in the Classification of Flood Disaster Grade," Arid Land Geography, 1999, pp. 37-42.

[6] G. Kim and A. P. Barros, "Quantitative Flood Forecasting Using Multisensor Data and Neural Networks," Journal of Hydrology, Vol. 246, No.1-4, 2001, pp. 45-62. doi:10.1016/S0022-1694(01)00353-5

[7] S.-J. Wang, X.-1. Zhang, Y. Hou and J. Ding, "Projection Pursuit Model for Evaluating of Flood Events," Hydrology, Vol. 22, 2002, pp. 1-4.

[8] A. Montanari and L. Lizzani, "A Projection Pursui Approach to Variable Selection," Computational Statisics \&
Data Analysis, Vol. 35, No. 4, 2001, pp. 463-473. doi:10.1016/S0167-9473(00)00026-8

[9] R. Poli, J. Kennedy and T. B. Well, "Particle Swarm Optimization: A Overview," Swarm Intelligence, Vol. 1, No. 1, 2007, pp. 33-57. doi:10.1007/s11721-007-0002-0

[10] J. V. Ast, R. Babuska and B. D. Schutter, "Particle Swarms in Optimization and Control," Proceedings of the 17th World Congress the International Federation of Automatic Control, Seoul, Korea, 6-11 July 2008, pp. 5131-5136.

[11] A. Banks, J. Vincent and C. Anyakoha, "A review of Particle Swarm Optimization. Part I: Background and Development," Natural Computing, Vol. 6, No. 4, 2007, pp. 467-484. doi:10.1007/s11047-007-9049-5

[12] J. Friedman and J. Tukey, "A Projection Pursuit Algorithm for Exploratory Data Analysis," Computers, Vol. 23, 1974, pp. 881-890.

[13] Y.-L. Lu, J.-Z. Zhou, L.-X. Song and Y.-Y. He, "Flood Disaster Evaluating Method Based on CCPSO along with Project Pursuit Model and Its Application," Journal of System Simulation, 2010, pp. 383-387+390.

[14] J. Kennedy and R. Eberhart, "Particle Swarm Optimization," IEEE International Conference Evolutionary Computation, Anchorage, 15-19 July 1995, pp. 19421948

[15] P. J. Angeline, "Evolutionary Optimization Versus Particle Swarm Optimization: Philosophy and Performance Difference," Proceedings of the 7th Annual Conference on Evolutionary Programming, San Diego, California, 26-29 March 1998, pp. 601-610. doi:10.1007/BFb0040811

[16] B. Niu, Y.-1. Zhu, X.-X. He and H. Shen, "A Multi-Swarm Optimizer Based Fuzzy Modeling Approach for Dynamic System Processing," Neurocomputing, Vol. 71, No.7-9, 2008, pp. 1436-1448. doi:10.1016/j.neucom.2007.05.010

[17] Y. Marinakis and M. Marinaki, "A Hybrid Multi-Swarm Particle Swarm Optimization Algorithm for the Probabilisitc Traveling Salesman Problem," Computers \& Operations Research, Vol. 37, No. 3, 2010, pp. 432-442. doi:10.1016/j.cor.2009.03.004

[18] J.-L. Jin, X.-L. Zhang, J. Ding, "Projection Pursuit Model for Evaluating Grade of Flood Disaster Loss," System Engineering-Theory \& Practice, 2002, pp. 140-144. 